\title{
Gross anatomical study on the gender differences in the corpus callosum
}

\author{
Isurani Ilayperuma ${ }^{1}$, Ganananda Nanayakkara $^{2}$, Nadeeka Palahepitiya $^{3}$ \\ ${ }^{1}$ Senior Lecturer in Anatomy, ${ }^{2}$ Professor in Anatomy, ${ }^{3}$ Technical Officer, Department of Anatomy, Faculty of \\ Medicine, University of Ruhuna, Galle. \\ e-mail address of the correspondingauthor,Prof.B.G.Nanayakkara: rosebudbgnn@yahoo.com
}

\begin{abstract}
Introduction: The corpus callosum of the mammalian brain is undoubtedly the most important commissure that connects the cerebral hemispheres due to the wealth of its neural connections. Much of the inter-hemispheric communication in the brain is conducted across the corpus callosum. However, the sexual dimorphism in the morphology of the corpus callosum has remained controversial.

Objective: This study was conducted to elucidate the gross anatomical differences in corpus callosum in a sample of Sri Lankan population.

Materials and Methods: A total of 34 formalin preserved human brains without apparent neuropathology were studied to ascertain sexual dimorphism in the human corpus callosum. Using mid-sagittal brain sections, several morphometric features of the corpus callosum were recorded: Straight distances between (i) frontal pole to occipital pole (ii) anterior most point to posterior most point of corpus callosum (iii) frontal pole of brain to anterior most point of corpus callosum (iv) occipital pole of brain to posterior most point of corpus callosum (v) anterior edge of splenium to superior most point of superior colliculus (vi) anterior edge of genu to anterior edge of columns of fornix.
\end{abstract}

Results: Statistically significant gender differences were observed between the mean values of all the parameters recorded.

Conclusion: Corpus callosum being the major structural connection between the cerebral hemispheres is likely to be affected by physiological as well as pathological changes in the cortical and sub-cortical regions of the brain. The precise anatomical knowledge regarding the morphology of corpus callosum as well as the gender related changes of the structure in a certain ethnic population will provide baseline data for the diagnosis and progression of a disease affecting it.

Key words: Corpus callosum, gender, Sri Lankan

\section{Introduction}

The corpus callosum is a structure in the longitudinal fissure of the mammalian brain that connects the left and right cerebral hemispheres. The posterior portion of the corpus callosum is called the splenium; the anterior is called the genu or "knee", between the two is the body of the corpus callosum. The rostrum is the portion of the corpus callosum that projects posteriorly following from the anterior most genu ${ }^{1,2}$.

The corpus callosum consists of a thick bundle of nerve fibers containing both myelinated and unmyelinated axons. It has been attributed the function of transfer of information between the two hemispheres. Each hemisphere contains neurons which project callosal axons not only to homologous areas in the contralateral hemisphere but also to heterologous areas. The corpus callosum is undoubtedly the most important commissure to connect the two hemispheres due to the wealth of its neural connections. Much of the inter-hemispheric communication in the brain is conducted across the corpus callosum ${ }^{3}$.

Very little is known about the neural signals that pass 
between the hemispheres. Recent studies have used modern tract tracing techniques to determine precisely the sites of origin and termination of neurons which project across the corpus callosum. Using a retrograde tracer (horseradish peroxidase), it was able to link the functional divisions of the cerebral cortex to fiber trajectory through the corpus callosum ${ }^{4}$.

In humans, disputed claims have been made about the gender difference in the morphometry of the corpus callosum ${ }^{5,6}$. Several studies have indicated significant sexual dimorphism in callosal structure $^{7-10}$ while some other studies reported the absence of such differences ${ }^{5,11}$. Most of the research on corpus callosum has been performed on Caucasian brains ${ }^{5,11,12}$ while very few on Asian population $^{13}$.

Despite its significance, little is known regarding the morphology and the Anatomical dimensions of the corpus callosum in Sri Lankans. Hence, this study was carried out to elucidate the gross anatomical features and gender differences in an adult Sri Lankan population.

\section{Materials \& Methods}

A total of 34 cadavers ( 22 males and 12 females) that was used for the routine gross anatomy dissections at the Department of Anatomy, Faculty of Medicine, Galle, Sri Lanka, were used for this study. Subjects were aged between 54 and 79 years.

The brains were removed from the cranial cavity and preserved in $10 \%$ formalin solutions. The brain specimens from people with intracranial lesions, head injury or recorded brain pathology were excluded from the study. Only those brains with no visible gross abnormalities were included in the study. Brains were sagittaly sectioned from front to back through the septum pellucidum.

Straight distances between the following structures were recorded:

(i) frontal pole to occipital pole (LB).

(ii) anterior most point to posterior most point of corpus callosum (LC).

(iii) frontal pole of brain to anterior most point of corpus callosum (FC).

(iv) occipital pole of brain to posterior most point of corpus calloum (OC). (v) anterior edge of splenium to superior most point of superior colliculus (SS).

(vi) anterior edge of genu to anterior edge of columns of fornix (GF).

The above measurements were recorded using a digital sliding caliper (Mitutoyo, Japan) capable of measuring to the nearest $0.01 \mathrm{~mm}$. All the measurements were repeated thrice and the mean was taken for further analysis. Furthermore, the measurements were recorded by the same person to minimize the errors in measurements. Results were expressed as mean $\pm \mathrm{SD}$. Data were analyzed using the Statistical Package for Social Sciences (SPSS), $15^{\text {th }}$ version. A comparison of the mean values between sides and genders was performed using the t-test. $\mathrm{P}$ value $<0.05$ was considered statistically significant.

\section{Results}

The results of the various parameters of the corpus callosum according to the gender are shown in Table 1 . Statistically significant $(\mathrm{P}$ value $<0.05)$ differences were observed between the mean values of all the parameters recorded. All the parameters were greater in males than in females.

Table 1 - Gender differences in various parameters of corpus callosum in human brains.

\begin{tabular}{|l|l|l|}
\hline Parameter (cm) & Male & Female \\
\hline LB & $16.02 \pm 0.86 *$ & $15.01 \pm 0.64$ \\
\hline LC & $8.03 \pm 0.64 *$ & $7.02 \pm 0.04$ \\
\hline FC & $3.42 \pm 0.46 *$ & $3.01 \pm 0.21$ \\
\hline OC & $5.74 \pm 0.62 *$ & $4.32 \pm 0.26$ \\
\hline SS & $0.96 \pm 0.14 *$ & $0.68 \pm 0.20$ \\
\hline GF & $3.10 \pm 0.42 *$ & $2.32 \pm 0.26$ \\
\hline
\end{tabular}

Data are expressed as mean \pm standard deviation.

* Pvalue $<0.05$.

\section{Discussion}

The present study provides new data concerning the morphology of corpus callosum in an adult Sri Lankan population. 
It is well known that human brain display sexual dimorphism $^{1,14}$. Besides the difference in human brain size, some cell groups of the hypothalamus, corpus callosum and other commissural systems display differences between the sexes ${ }^{14}$. Further, dimorphisms involve the temporal lobes and sexspecific brain asymmetry ${ }^{14}$. However, the results obtained for the corpus callosum and other commissural systems, are inconclusive ${ }^{5-10}$.

During developmental period volumes of the cerebrum, lateral ventricles, caudate, putamen, temporal lobe, hippocampus, and mid-sagittal area measurements of the corpus callosum are known to exert gender differences ${ }^{15}$. From clinical standpoint of view, these sexually dimorphic patterns of brain development may be related to the observed sex differences in age of onset, prevalence, and symptomatology seen in nearly all neuropsychiatric disorders of childhood ${ }^{15}$.

It is often argued that gender differences in human brain morphology may have an impact on cognitive gender differences. Various methods such as cadaver studies and magnetic resonance imaging (MRI) of brain have been used to study the morphology and anatomical dimensions of the corpus callosum ${ }^{5}$. These studies have confirmed the earlier findings of larger average brain size and overall corpus callosum size for males. The observations of the present study, statistically significant gender differences observed in length of brain, length of corpus callosum and these parameters being greater in males than in females, were in agreement with the above findings ${ }^{7-10}$. Furthermore, our results highlight the findings of our results are in agreement with the observations of Suganthy et al., $2003^{13}$.

Recent studies using new techniques revealed morphological sex differences in human corpus callosum ${ }^{16,17,18}$. These structural changes could be the basis of gender related changes in certain cognitive functions of the brain. Whether and to what extent, these morphological differences are associated with behavioural and cognitive differences between men and women remains unclear.

Corpus callosum being the major structural connection between the hemispheres is likely to be affected by physiological as well as pathological changes in the cortical and sub-cortical regions of the brain. The fiber systems that connects the corresponding cerebral hemispheres travels through specific regions of the corpus callosum. In severe epileptic patients when drug treatment is ineffective, surgical intervention on reducing the interhemispheric transmission of abnormal electrical discharges involves the sectioning of the corpus callosum and other commissural structures ${ }^{19}$. Hence, any morphological changes in corpus callosum may give a clue which will help diagnosis of specific pathological condition.

The precise anatomical knowledge regarding the morphology of corpus callosum as well as the gender related changes of the structure in a certain ethnic population will provide baseline data for the diagnosis and assessment of progression of a disease affecting it.

\section{Acknowledgement}

The authors wish to thank Dr. W.A.A. Wijayasiri, Department of Community Medicine, Faculty of Medicine, University of Ruhuna, Galle, Sri Lanka, for advising with the statistical analysis.

\section{References}

1. Williams PL, Bannister LH, Berry MM, Collins P, Dyson M, Dussek JE. Gray's Anatomy: The Anatomical Basis of Medicine and Surgery. $38^{\text {th }}$ Ed. Churchill Livingstone, 2000.

2. Sinnathamby CS. Last's Anatomy Regional and Applied. $10^{\text {th }}$ Ed. Edinburgh: Churchill Livingstone, 1999.

3. Snell R. Clinical Neuroanatomy. $7^{\text {th }}$ Edn. Churchill Livingstone, 2000.

4. Lomber SG, Payne BR, Rosenquist AC. The spatial relationship between the cerebral cortex and fiber trajectory through the corpus callosum of the cat. Behavioural Brain Research 1994; 64: 25-35.

5. Bishop KM, Wahlsten D. Sex differences in the human corpus callosum: myth or reality? Neuroscience Biobehavioural Review 1997; 21: 581-601.

6. Constant D, Ruther H. Sexual dimorphism in the human corpus callosum? A comparison of methodologies. Brain Research 1996; 727: 99-106.

7. DeLacoste C, Holloway RL. Sexual dimorphism in the human corpus callosum. Science 1982; 216: 1431-2. 
8. Going JJ, Dixson A. Morphometry of the adult human corpus callosum: lack of sexual dimorphism. Journal of Anatomy 1990; 171: 163-7.

9. Holloway RL, Anderson PJ, Defendini R, Harper C. Sexual dimorphism of the human corpus callosum from three independent samples: relative size of the corpus callosum. American Journal of Physical Anthropology 1993; 92: 481-98.

10. Allen LS, Richey MF, Chai YM, Gorski RA. Sex differences in the corpus callosum of the living human being. Journal of Neuroscience 1991; 11: 933-42.

11. Witelson SF. Hand and sex differences in the isthmus and genu of the human corpus callosum: A postmortem morphological study. Brain 1989; 112: 799-835.

12. Luders E, Rex DE, Narr KL, Woods RP, Jancke L, Thompson PM, Mazziotta JC, Toga AW. Relationships between sulcal asymmetries and corpus callosum size: gender and handedness effects. Cerebral Cortex 2003; 13: 1084-93.

13. Suganthy J, Raghuram L, Antonisamy B. Gender and age related differences in the morphology of corpus callosum. Clinical Anatomy 2003; 16:396-403.

14. Supprian T, Kalus P. Sexual dimorphism of the human brain-a review of the literature. Fortschritte der Neurologie-Psychiatrie 1996; 64: 382-9. 